\title{
Magnetic studies reveal near-perfect paramagnetism in the molecular semiconductor vanadyl phthalocyanine $\left(\mathrm{C}_{32} \mathrm{H}_{16} \mathrm{~N}_{8} \mathrm{VO}\right)$
}

Zhengjun Wang (王正君 $)^{1}, \mathrm{Li} \mathrm{Pi}^{2}$, Mohindar S. Seehra ${ }^{1}$, Jasleen Bindra ${ }^{3}$, Hans van $\mathrm{Tol}^{3}$ and Naresh S. Dalal ${ }^{3}$

${ }^{1}$ Department of Physics and Astronomy, West Virginia University, Morgantown, WV 265066315

${ }^{2}$ High Magnetic Field Laboratory, Chinese Academy of Sciences and University of Science and Technology of China, Hefei 230031, China

${ }^{3}$ National High Magnetic Field Laboratory, and Department of Chemistry and Biochemistry, Florida State University, Tallahassee, FL 32306

\begin{abstract}
Temperature $(0.5 \mathrm{~K}$ to $300 \mathrm{~K}$ ) and magnetic field ( $\mathrm{H}$ up to $90 \mathrm{kOe}$ ) dependences of the magnetization (M) of a powder sample of vanadyl phthalocyanine (VOPc) having the Phase IItriclinic structure are measured and analyzed. The data of $\chi=\mathrm{M} / \mathrm{H}$ vs. T measured in $\mathrm{H}=1 \mathrm{kOe}$ fit the modified Curie-Weiss (CW) law, $\chi=\chi_{0}+\mathrm{C} /(\mathrm{T}-\theta)$, with $\mathrm{C}=6.266 \times 10^{-4} \mathrm{emuK} / \mathrm{gOe}, \theta=$ - $0.1 \mathrm{~K}$ and $\chi_{\mathrm{o}}=-9.3 \times 10^{-7} \mathrm{emu} / \mathrm{gOe}$. The Curie constant $\mathrm{C}$ yields magnetic moment $\mu=1.704 \mu_{\mathrm{B}}$, $\mathrm{S}=1 / 2$, and $\mathrm{g}=1.967$ characteristic of $\mathrm{VO}^{2+}$. The magnitude of $\theta=-0.1 \mathrm{~K}$ signifying very weak inter-ion antiferromagnetic exchange coupling is supported by the analysis of the variable frequency $(9.8$ - $336 \mathrm{GHz})$ electron paramagnetic resonance data. The isothermal data of $\mathrm{M}$ vs. $\mathrm{H}$ at ten temperatures between $0.5 \mathrm{~K}$ and $300 \mathrm{~K}$ when plotted as $\mathrm{M}$ vs. $\mathrm{H} /(\mathrm{T}+0.1)$ collapses on to a single curve given by $\mathrm{M}=\mathrm{M}_{\mathrm{o}} \tanh \left\{\mathrm{g} \mu_{\mathrm{B}} \mathrm{H} /\left[2 \mathrm{k}_{\mathrm{B}}(\mathrm{T}+0.1)\right]\right\}$ with $\mathrm{M}_{\mathrm{o}}=\mathrm{Ng} \mu_{\mathrm{B}} \mathrm{S}=9.48 \mathrm{emu} / \mathrm{g}$ expected for $S=1 / 2$ system, thus signifying near perfect paramagnetism in VOPc.
\end{abstract}

Key words: Magnetic susceptibility, Paramagnetism, Electron paramagnetic resonance, Saturation magnetization, Magnetic molecular semiconductors.

*Corresponding author. Email: mseehra@wvu.edu; Phone: 304-293-5098. 


\section{Introduction:}

Transition-metal-substituted phthalocyanines (TMPc, TM = Mn, Fe, Co, Ni, and $\mathrm{Cu}$ ) with the general formula of $\mathrm{C}_{32} \mathrm{H}_{16} \mathrm{~N}_{8} \mathrm{TM}$ are organic semiconductors with interesting photoconductive properties and have potential applications in optoelectronic devices [1]. Structurally, these TMPc are planar molecules with the TM atom at the center bound to four $\mathrm{N}$ atoms and forming a linear chain along the monoclinic b-axis. Recent magnetic studies reported in $\mathrm{CuPc}, \mathrm{CoPc}$, and $\mathrm{MnPc}$ show that the exchange coupling between the TM ions is either ferromagnetic as in $\operatorname{MnPc}[2,3]$ or antiferromagnetic as in $\mathrm{CuPc}[4]$ and $\mathrm{CoPc}[5,6]$. In contrast to $\mathrm{CuPc}, \mathrm{CoPc}$, and $\mathrm{MnPc}$, VOPc is a nonplanar molecule and has a five- coordinate square pyramidal structure with a single electron associated with $\mathrm{VO}^{2+}$ ion [7]. Analysis of the x-ray diffraction data by Ziolo et al [8] showed that $\mathrm{V}$ atom is $0.0575 \mathrm{~nm}$ above the molecular plane with a $\mathrm{V}-\mathrm{O}$ distance $=0.1580 \mathrm{~nm}$ and the unit cell is triclinic with two molecules per unit cell (Figure 1). Besides the common use as an organic pigment, VOPc also shows potential applications in high performance thin-film transistors [9].

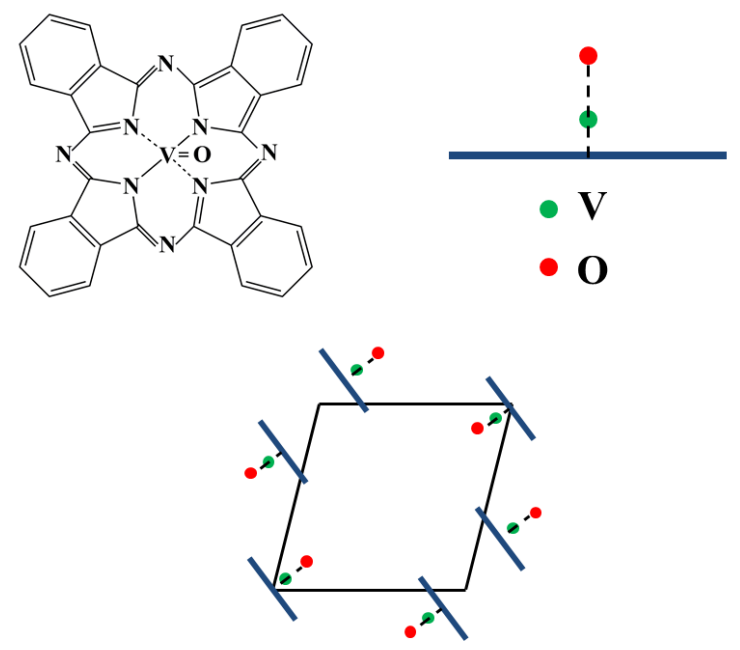

Fig. 1. Molecular structure and unit cell of VOPc (phase II). The lower panel shows a view along the b-axis of the unit cell. The blue lines in the unit cell depict the orientation of the molecular planes of VOPc. 
Regarding the nature of magnetism of VOPc, recent studies report it to be a paramagnet [10] based on the earlier work of Lever [11] which effectively list its magnetic moment $\mu=$ $1.73 \mu_{\mathrm{B}}$ corresponding to a free electron, $\mu_{\mathrm{B}}$ being the Bohr magneton. However, temperature and magnetic field dependence of its magnetization has not been reported to verify such a designation. In order to understand the nature of magnetism of VOPc, we have carried out measurements of the magnetization $\mathrm{M}$ of a powder sample of $\mathrm{VOPc}$ as a function of temperature $\mathrm{T}$ from $0.5 \mathrm{~K}$ to $300 \mathrm{~K}$ in magnetic field $\mathrm{H}$ up to $90 \mathrm{kOe}$. The $\chi$ vs. T data are shown to fit well to the modified Curie-Weiss $(\mathrm{CW})$ law giving $\theta=-0.1 \mathrm{~K}, \mu=1.704 \mu_{\mathrm{B}}, \mathrm{S}=1 / 2$, and $\mathrm{g}=1.967$ for $\mathrm{VO}^{2+}$. This magnitude of $\theta=-0.1 \mathrm{~K}$ indicates very weak antiferromagnetic exchange coupling among the $\mathrm{VO}^{2+}$ ions in contrast to other TMPc systems such as MnPc, CuPc, and CoPc, in which much stronger exchange coupling between the TM ions has been reported [2-6]. Our M vs. H isothermal data in VOPc at different temperatures between $0.5 \mathrm{~K}$ and $300 \mathrm{~K}$ is found to fit well with the Brillouin function variation for spin $S=1 / 2$ with exchange coupling $\theta=-0.1 \mathrm{~K}$ taken into account. This confirms the near-perfect paramagnetism of VOPc but with some expected and notable orbital contribution to the magnetic moment. Analysis of the variable frequency $(9.8$ $\mathrm{GHz}$ to $336 \mathrm{GHz}$ ) electron paramagnetic resonance (EPR) measurements are also reported which supports the above conclusion of near perfect paramagnetism. Details of these results, their analysis and discussion are given below.

2. Sample and its structural characterization: The powder sample of VOPc was purchased from Sigma Aldrich (stock \# 791997) and used as received. The X-ray diffraction (XRD) of this sample was performed using PANalytical X'Pert Pro X-ray diffractometer with monochromatic $\mathrm{Cu}-\mathrm{K} \alpha$ radiation of wavelength $\lambda=0.15418 \mathrm{~nm}$. The XRD pattern of the sample is shown in Fig. 2 along with the Miller indices of the major Bragg lines. The position of the Bragg lines were calculated using the parameters listed below and it was found that the structure matched perfectly with that of phase II for VOPc having space group $\mathrm{P} \overline{1}$ with two molecules per unit cell of the 
triclinic structure and lattice parameters: $\mathrm{a}=1.2027 \mathrm{~nm}, \mathrm{~b}=1.2571 \mathrm{~nm}, \mathrm{c}=0.8690 \mathrm{~nm}, \alpha=$ $96.04^{\circ}, \beta=94.80^{\circ}$, and $\gamma=68.20^{\circ}[6]$. This structure is depicted in Fig. 1 where it is noted that unlike the other TMPc systems such as $\mathrm{CuPc}, \mathrm{CoPc}$ and MnPc, VOPc is a non-planar molecule having a five-coordinate square pyramidal structure. Using the calculated volume of the unit cell $=1.2117 \times 10^{-21} \mathrm{~cm}^{3}$ with two molecules per unit cell and a single unpaired electron associated with each $\mathrm{VO}^{2+}$ ion, $\mathrm{n}=1.65 \times 10^{21}$ per $\mathrm{cm}^{3}$ is determined as the number of spins per unit volume. This information is used later in this paper.

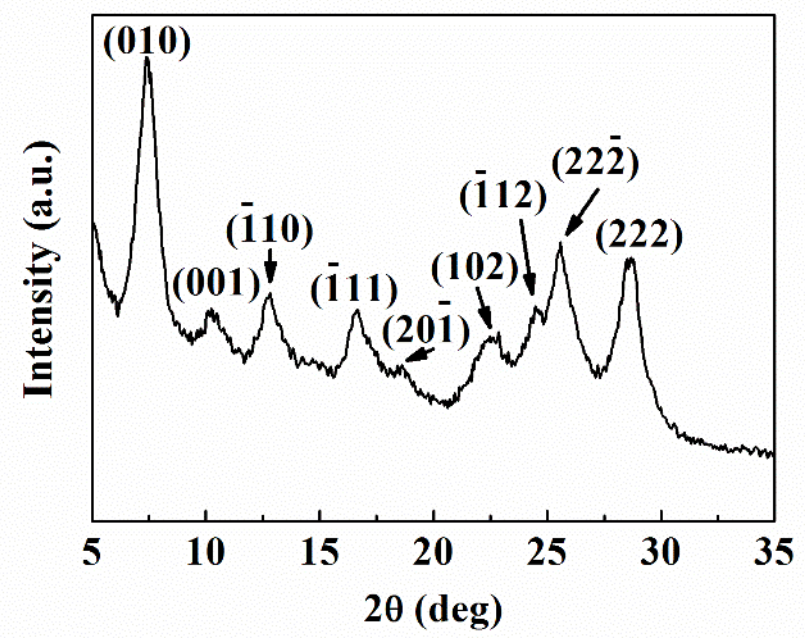

Fig. 2. XRD pattern of the VOPc powder sample with Miller Indices of major peaks shown. No impurity lines were observed.

\section{Experimental results and analysis:}

3.1. Temperature dependence of magnetization : Measurements of the magnetization $M$ as a function of temperature $\mathrm{T}$ from $2 \mathrm{~K}$ to $300 \mathrm{~K}$ and in magnetic field $\mathrm{H}$ up to $90 \mathrm{kOe}$ were performed at WVU using a physical property measurement system (PPMS) manufactured by Quantum Design Inc. Additional M vs. T data from $0.5 \mathrm{~K}$ to $2 \mathrm{~K}$ and $\mathrm{M}$ vs. $\mathrm{H}$ up to $70 \mathrm{kOe}$ at 0.5 $\mathrm{K}, 1 \mathrm{~K}$, and $1.5 \mathrm{~K}$ were obtained at the High Magnetic Field Laboratory, Chinese Academy of Sciences (CHMFL) using a magnetic property measurement system (MPMS) manufactured by 
Quantum Design Inc. Fig. 3 shows the combined data of the temperature dependence of magnetic susceptibility $\chi=\mathrm{M} / \mathrm{H}$ for VOPc powder sample measured at $\mathrm{H}=1 \mathrm{kOe}$. The solid lines are fits to the modified Curie-Weiss $(\mathrm{CW})$ law $: \chi=\chi_{0}+\mathrm{C} /(\mathrm{T}-\theta)$, where $\chi_{0}=-9.3 \times 10^{-7}$ $\mathrm{emu} / \mathrm{gOe}$ is the diamagnetic contribution estimated from the high-temperature limit by plotting $\chi$ vs. $1 / \mathrm{T}$ and extrapolating to the limit $1 / \mathrm{T}=0$. Note that $\chi_{\mathrm{o}}$ contains diamagnetic contributions of both VOPc as well as the plastic sample holder used in the measurements. However, because of its comparatively small magnitude, the effects of $\chi_{0}$ are only noticeable at the higher temperatures. The data of M vs. H shown later was also corrected for $\chi_{\mathrm{o}}$

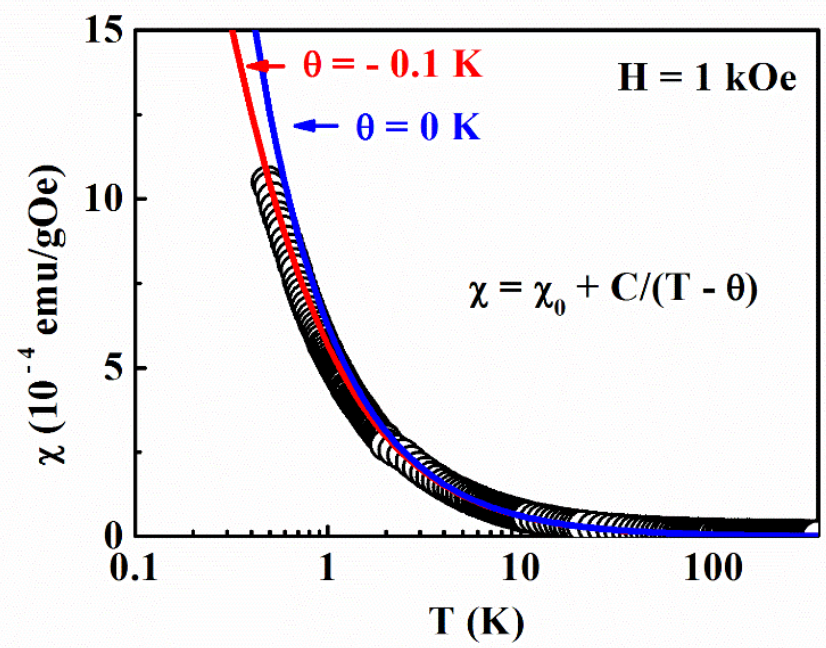

Fig. 3. Magnetic susceptibility $\chi$ versus temperature $\mathrm{T}$ from $0.5 \mathrm{~K}$ to $300 \mathrm{~K}$ of the VOPc powder sample. with $\mathrm{H}=1 \mathrm{kOe}$. The open circles are experimental data and the solid lines are fit to the modified Curie-Weiss law with the parameters: $\chi_{0}=-9.3 \times 10^{-7} \mathrm{emu} / \mathrm{gOe}, \mathrm{C}=6.266 \times 10^{-4}$ emuK/gOe, and $\theta=-0.1 \mathrm{~K}$ and $0 \mathrm{~K}$. 


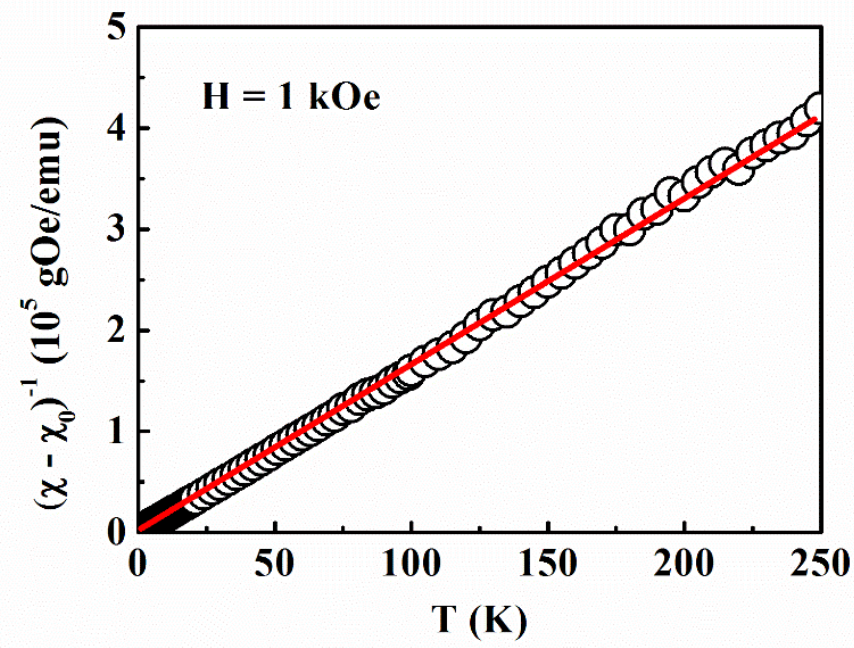

Fig. 4. The data of Fig. 3 plotted as $\left(\chi-\chi_{0}\right)^{-1}$ vs. T. The solid line is the linear fit.

After determining $\chi_{0}$, the data of $\left(\chi-\chi_{0}\right)^{-1}$ was plotted against temperature (Fig.4) yielding a straight line whose slope $=1 / \mathrm{C}$ yielded $\mathrm{C}=6.266 \times 10^{-4} \mathrm{emuK} / \mathrm{gOe}$. The intercept on the T-axis yields $\theta=-0.1 \mathrm{~K}$ within the experimental uncertainty. The solid lines in Fig. 3 are fits to the modified CW law shown for both $\theta=-0.1 \mathrm{~K}$ and $\theta=0 \mathrm{~K}$. It is evident that the choice of $\theta=-0.1$ $\mathrm{K}$ provides a better fit to the data especially for $\mathrm{T}<2 \mathrm{~K}$. This magnitude of $\theta=-0.1 \mathrm{~K}$ represents a very weak antiferromagnetic exchange coupling between the $\mathrm{VO}^{2+}$ ions and the so near-perfect paramagnetic nature of VOPc. In comparison, in the corresponding systems of $\alpha$-CuPc and $\beta$ $\mathrm{CuPc}$ [4] and $\beta$-CoPc [6], all with $\mathrm{S}=1 / 2$, considerably larger magnitudes of $\theta=-2.3 \mathrm{~K},-0.2 \mathrm{~K}$ and $-2.5 \mathrm{~K}$ respectively were observed. Assuming a linear chain antiferromagnetic system as in $\alpha-\mathrm{CuPc}$ and $\beta$-CuPc [4] and $\beta$-CoPc [6], yields $\theta=1.297 \mathrm{~J} / \mathrm{k}_{\mathrm{B}}$ where $\mathrm{J}$ is the Heisenberg exchange constant in the Hamiltonian $\hat{\mathrm{H}}=-2 \mathbf{J} \sum \mathbf{S}_{\mathbf{i}} \mathbf{\bullet} \mathbf{S}_{\mathbf{i + 1}}$ and $\mathrm{k}_{\mathrm{B}}$ is the Boltzmann constant. This analysis yields $\mathrm{J} / \mathrm{k}_{\mathrm{B}}=-0.077 \mathrm{~K}$ as the exchange coupling constant in VOPc for $\theta=-0.1 \mathrm{~K}$. Also, knowing that $\mathrm{C}=\mathrm{N} \mu^{2} / 3 \mathrm{k}_{\mathrm{B}}$ where $\mathrm{N}$ is the number of magnetic ion per gm and $\mu$ is the magnetic moment per ion, the evaluated $\mathrm{C}=6.266 \times 10^{-4} \mathrm{emuK} / \mathrm{gOe}$ yields magnetic moment $\mu=1.704 \mu_{\mathrm{B}}$ 
which for $S=1 / 2$, yields $g=1.967$ by using $\mu^{2}=g^{2} S(S+1) \mu_{B}^{2}$. The magnitude of $g<2$ due to orbital contribution is expected for TM ions with d-shell less than half full as is valid for $\mathrm{VO}^{2+}$.

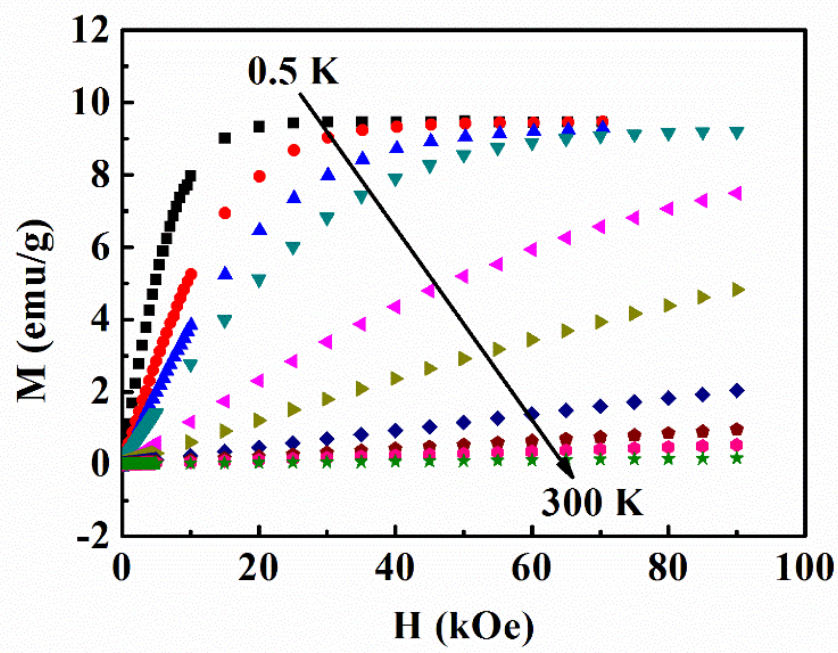

Fig. 5. Magnetization $M$ vs. magnetic field $H$ data for the VOPc powder at $0.5 \mathrm{~K}, 1 \mathrm{~K}, 1.5 \mathrm{~K}, 2 \mathrm{~K}$, $5 \mathrm{~K}, 10 \mathrm{~K}, 25 \mathrm{~K}, 50 \mathrm{~K}, 100 \mathrm{~K}$, and $300 \mathrm{~K}$. Note that the data at $0.5 \mathrm{~K}, 1 \mathrm{~K}$, and $1.5 \mathrm{~K}$ are with $\mathrm{H}$ up to $70 \mathrm{kOe}$; data at other temperatures are with $\mathrm{H}$ up to $90 \mathrm{kOe}$.

3.2 Magnetic field dependence of magnetization: The isothermal data of $\mathrm{M}$ vs. $\mathrm{H}$ up to $90 \mathrm{kOe}$ measured at $0.5 \mathrm{~K}, 1 \mathrm{~K}, 1.5 \mathrm{~K}, 2 \mathrm{~K}, 5 \mathrm{~K}, 10 \mathrm{~K}, 25 \mathrm{~K}, 50 \mathrm{~K}, 100 \mathrm{~K}$, and $300 \mathrm{~K}$ for VOPc are shown in Fig. 5. To verify the near-perfect paramagnetism of VOPc, the isothermal data of M vs. $\mathrm{H}$ for all temperatures is plotted in terms of $\mathrm{M}$ vs. $\mathrm{H} /(\mathrm{T}-\theta)$ in Fig. 6 to check its Brillouin function variation expected for $S=1 / 2$ systems given by [12]:

$$
M=M_{o} \tanh \left[\frac{g \mu_{B} H}{2 k_{B}(T-\theta)}\right]
$$

This plot in Fig. 6 shows collapsing of all the data on to a single curve which fits Eq. (1) very well with $\theta=-0.1 \mathrm{~K}$ implying the very weak antiferromagnetic exchange coupling between the $\mathrm{VO}^{2+}$ ions. Obviously the effect of including $\theta=-0.1 \mathrm{~K}$ in only meaningful for temperatures below $2 \mathrm{~K}$ signifying the importance of these low temperature investigations in this case. Notice that saturation magnetization $\mathrm{M}_{\mathrm{o}}=9.46 \mathrm{emu} / \mathrm{g}$ at $0.5 \mathrm{~K}$ agrees well with the calculated value of 
$\mathrm{M}_{\mathrm{o}}=9.48 \mathrm{emu} / \mathrm{g}$ using $\mathrm{M}_{\mathrm{o}}=\mathrm{Ng} \mu_{\mathrm{B}} \mathrm{S}$ with $\mathrm{g}=1.967$ and $\mathrm{S}=1 / 2$. The molecular weight $(\mathrm{MW})=$ 579.46 for VOPc is used in these calculations to determine $\mathrm{N}=\mathrm{N}_{\mathrm{A}} / \mathrm{MW}$ where $\mathrm{N}_{\mathrm{A}}$ is the Avogadro's number.

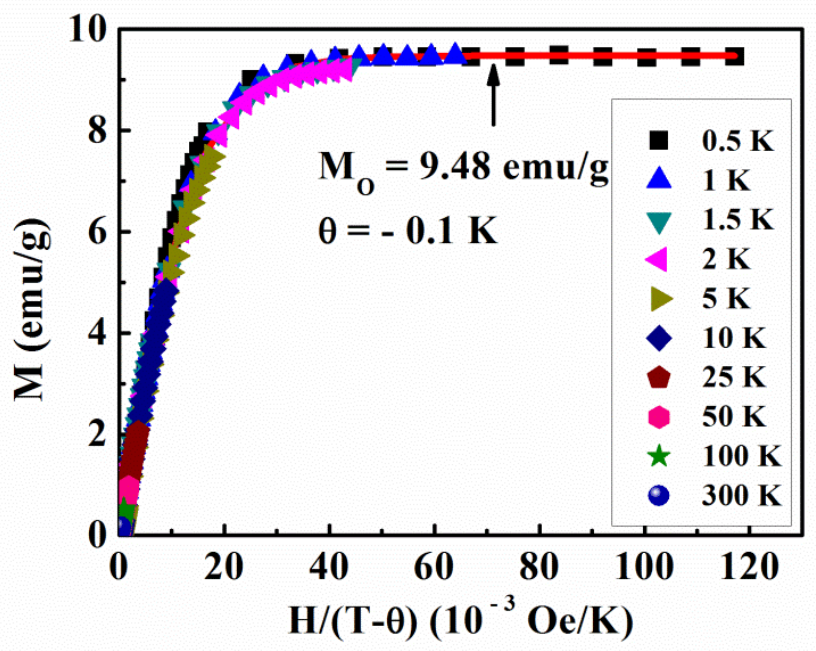

Fig. 6. Plot of the data of Fig. 5 in terms of $M$ vs. $H /(T-\theta)$ with $\theta=-0.1 \mathrm{~K}$. The solid line (red color) is the fit to Eq. 1 with $\mathrm{g}=1.967, \mathrm{M}_{\mathrm{o}}=9.48 \mathrm{emu} / \mathrm{g}$ and $\theta=-0.1 \mathrm{~K}$.

3.3. Electron Paramagnetic Resonance (EPR): EPR is a complementary technique to magnetic measurements to accurately determine the g-value, and in particular, to detect the presence of spin exchange interaction in the system. The reported EPR studies in VOPc so far are as follows: EPR in powder VOPc [13] showing a broad absorption near $\mathrm{g}=2$ with linewidth $\Delta \mathrm{H} \sim 500$ Oe; and (ii) papers by Assour et al. [14] and Sato et al. [15] on X-band ( 9.5 GHz) EPR studies in VOPc diluted (1:1000) in $\mathrm{H}_{2} \mathrm{SO}_{4}, \mathrm{H}_{2} \mathrm{Pc}$ and $\mathrm{ZnPc}$ which allowed them to observe the eight hyperfine lines corresponding to the nuclear spin $I=7 / 2$ of the $V^{51}$ nucleus and electron spin $S=1 / 2$ associated with $\mathrm{VO}^{2+}$. Two sets of the eight lines were observed, one set corresponding to $g_{\|}=$ 1.965 and the other set corresponding to $g_{\perp}=1.988$. Consequently, the EPR spectrum was fitted to the following standard Hamiltonian with axial symmetry including the hyperfine interaction:

$$
\mathcal{H}=g_{\perp} \mu_{B}\left(H_{x} S_{x}+H_{y} S_{y}\right)+g_{\|} \mu_{B} H_{z} S_{z}+A_{\|} I_{z} S_{z}+A_{\perp}\left(I_{x} S_{x}+I_{y} S_{y}\right)
$$


Here, $g_{\|}$and $A_{\|}$are in the direction of the $\mathrm{V}=\mathrm{O}$ axis of VOPc and $g_{\perp}$ and $A_{\perp}$ are in the direction perpendicular to it. The fitted parameters were: $g_{\|}=1.965$ and $\mathrm{g}_{\perp}=1.988$ yielding an average $\mathrm{g}=$ 1.98, and $\mathrm{V}^{51}$ hyperfine components $A_{\|}=160 \mathrm{G}$ and $A_{\perp}=60 \mathrm{G}$, It is noted here that the dilution of the sample was done to eliminate the dipole-dipole and exchange couplings between the electron spins which then allows the observation of the hyperfine structure.

In our EPR experiments, we used the same undiluted sample of powder VOPc as used in magnetic measurements. However the important new contribution of this work is that the data were taken over a range of frequencies from $9.8 \mathrm{GHz}$ to $336 \mathrm{GHz}$ in order to access information on the effects of anistropies due to g-values and hyperfine interactions in the presence of dipoledipole and exchange interaction, the latter indicated in the magnetic studies. The experiments were carried out at room temperature $(290 \mathrm{~K})$ using a commercial Bruker (X and Q-band) spectrometer as well as the high frequency $(240 \mathrm{GHz}$ and $336 \mathrm{GHz})$ spectrometers available at the National High Magnetic Field Laboratory in Tallahassee, Florida.
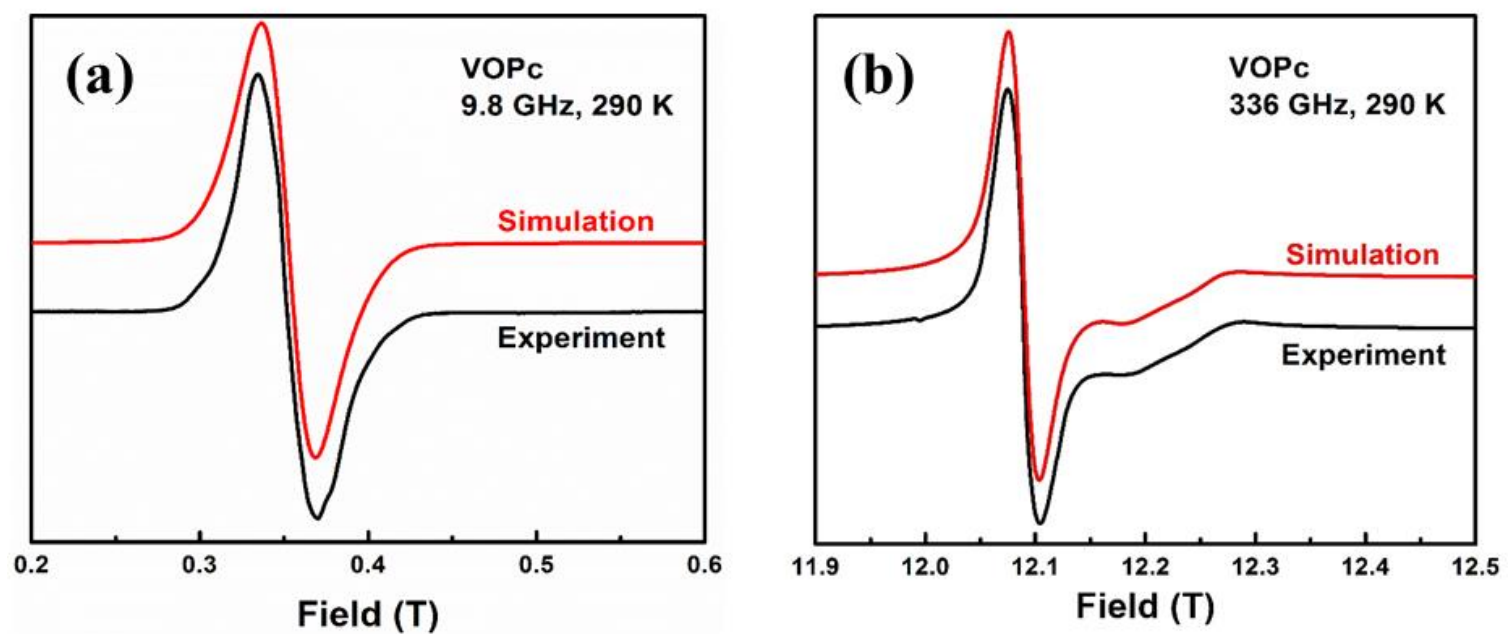

Fig. 7. Room temperature $9.8 \mathrm{GHz}$ and $336 \mathrm{GHz}$ EPR spectra of VOPc (black) and the best fit simulation (red) using the parameters $\mathrm{S}=1 / 2, \mathrm{I}=7 / 2, g_{\perp}=1.9844, g_{\|}=1.9631, A_{\perp}=17 \mathrm{G}$, and $A_{\|}=113 \mathrm{G}$. 
The observed X-band (9.8 GHz) EPR spectrum of the VOPc powder in Fig. 7(a) shows only a single line with a peak-to-peak width $\Delta \mathrm{H}=357 \pm 1 \mathrm{G}$ similar to the earlier studies reported by Ingram et al. [13]. The spectra at $240 \mathrm{GHz}$ (not shown) and at $336 \mathrm{GHz}$ shown in Fig. 7(b) are more revealing in that the single peak now splits into two peaks, as expected for a pancakeshaped molecule. Its most noteworthy feature is that it shows well resolved parallel and perpendicular peaks, but without any hyperfine structure. The width of the parallel peak at the higher fields in Fig. 7(b) is about 500 Oe. These spectra were analyzed using a locally developed computer program, EPRCALC, which diagonalizes the Hamiltonian matrix in Eq. (2) and uses the linewidth as a fitting parameter $[16,17]$. The spectra at $9.8 \mathrm{GHz}$ and $336 \mathrm{GHz}$ frequencies were well fit using the parameters $\mathrm{S}=1 / 2, \mathrm{I}=7 / 2, g_{\perp}=1.9844, g_{\|}=1.9631, A_{\perp}=17 \mathrm{Oe}, A_{\|}=$ $113 \mathrm{Oe}$, and variable linewidths. Note that in this undiluted VOPc, the hyperfine coupling $A_{\|}=$ 113 Oe is significantly smaller than $A_{\|}=160 \mathrm{G}$ observed for the diluted solutions of VOPc [14, 15]. This can be attributed to partial motional averaging combined with the decrease in width due to electron exchange along the $\mathrm{V}=\mathrm{O}$ direction. Also, although hyperfine energy terms were necessary to obtain good agreement between our simulation and experimental spectra, hyperfine splitting was not evident in these spectra possibly due to the presence of dipole-dipole and exchange coupling between to the $\mathrm{VO}^{2+}$ spins as discussed in the next section. The line-shape observed at X-band was analyzed and it was found to be only partially $(30-40 \%)$ Lorentzian.

\section{Discussion:}

In general, all anisotropies can broaden the EPR line in a powder sample whereas Heisenberg exchange interaction narrows the line [18] and the exchange narrowed lines are usually Lorentzian except perhaps in the tails of the lines [19]. In addition to the dipole-dipole interaction, other sources of line broadening include unresolved g-value anisotropy and unresolved hyperfine interaction [20]. Comparing the EPR linewidth observed in the powder $\operatorname{VOPc}$ at $9.8 \mathrm{GHz}(\Delta \mathrm{H}=360 \mathrm{Oe})$ with that observed at $336 \mathrm{GHz}\left(\Delta \mathrm{H}=290 \mathrm{Oe}\right.$ for $\left.g_{\perp}\right)$, it is 
inferred that the measured $\Delta \mathrm{H}=290$ Oe for the $g_{\perp}$ component does not have any broadening due to g-value anisotropy (Fig. $7 \mathrm{~b}$ ). Since $\Delta \mathrm{H}=500$ Oe for the $g_{\|}$component, it also follows that the linewidths due to $g_{\|}$and $g_{\perp}$ components cannot be simply added to yield the unresolved $\Delta \mathrm{H}=$ 360 Oe measured at $9 \mathrm{GHz}$. Similar argument is likely valid when considering the inhomogeneous broadening due to unresolved hyperfine interaction.

The exchange narrowing of the dipole-dipole broadened line can be estimated using the Anderson-Weiss theory [18] according to which $(\delta \mathrm{H})_{1 / 2}=\mathrm{H}_{\mathrm{p}}{ }^{2} / \mathrm{H}_{\mathrm{ex}}$ with

$$
\begin{aligned}
& H_{p}^{2}=5.1\left(g \mu_{B} n\right)^{2} S(S+1) \\
& H_{e x}=\frac{1.68 J[S(S+1)]^{1 / 2}}{g \mu_{B}}
\end{aligned}
$$

Here $\mathrm{n}$ is the number of spins $/ \mathrm{cm}^{3}$, and $(\delta \mathrm{H})_{1 / 2}$ is the half-width at half-maximum linewidth. Eq. (4) is valid for a cubic system with $Z=6$ as the number of nearest neighbor with significant exchange coupling $\mathbf{J}$ in the Heisenberg exchange interaction. For VOPc, $Z=2$ is more appropriate as noted earlier making $\mathrm{H}_{\mathrm{ex}}$ reduced by a factor of 3 from that given by Eq.(4). Further, using $\mathrm{n}=1.65 \times 10^{21} / \mathrm{cm}^{3}$ (see section 2$), \mathrm{J} / \mathrm{k}_{\mathrm{B}}=0.077 \mathrm{~K}$ and $\mathrm{S}=1 / 2$ determined from magnetic measurements, magnitudes of $\mathrm{H}_{\mathrm{p}}=59.2$ Oe and $\mathrm{H}_{\mathrm{ex}}=281$ Oe are calculated for VOPc. These magnitudes yield calculated $(\delta \mathrm{H})_{1 / 2}=\mathrm{H}_{\mathrm{p}}{ }^{2} / \mathrm{H}_{\mathrm{ex}}=12.5$ Oe. This calculated linewidth for exchange-narrowed and dipolar-broadened line is considerably smaller than the measured peakto-peak linewidth $\Delta \mathrm{H}=290$ Oe for $g_{\perp}$, even when the relationship $(\delta \mathrm{H})_{1 / 2}=0.59 \Delta \mathrm{H}(0.866 \Delta \mathrm{H})$ for Gaussian (Lorentzian) linewidths are taken into account. Also, it is noted that the "10/3" effect broadening of the exchange-narrowed dipolar linewidth $[18,21]$ often observed in systems when $\mathrm{H}_{\mathrm{ex}} \gg \mathrm{H}$ (applied) is not applicable for VOPc since calculated $\mathrm{H}_{\mathrm{ex}}=281 \mathrm{Oe}$ is much smaller than $\mathrm{H} \approx 3500$ Oe needed to observe the EPR line even at $9.8 \mathrm{GHz}$. Therefore it is concluded that inhomogeneous broadening due to unresolved hyperfine interaction in the powder 
sample is the likely major source of the observed linewidth. However as shown in our example of line-broadening due to g-value anisotropy in Fig. 7, this broadening due to unresolved hyperfine interaction cannot be quantitatively estimated using our measured values of $A_{\|}=113$ Oe and $A_{\perp}$ $=17$ Oe. The fact that the observed line-shape is only partially Lorentzian suggests the presence

of small yet non-negligible exchange coupling between the $\mathrm{VO}^{2+}$ spins as determined here from the analysis of the $\mathrm{M}$ vs. $\mathrm{T}$ and $\mathrm{M}$ vs. $\mathrm{H}$ data.

\section{Conclusions:}

The excellent fit of $\chi$ vs. $\mathrm{T}$ data from $0.5 \mathrm{~K}$ to $350 \mathrm{~K}$ in VOPc to the modified Curie-Weiss law yielding $\theta=-0.1 \mathrm{~K}$ represents very weak antiferromagnetic exchange coupling between the $\mathrm{VO}^{2+}$ ions for this spin $\mathrm{S}=1 / 2$ system, implying near-perfect paramagnetism. This conclusion is confirmed by the observed collapse of the isothermal data of $\mathrm{M}$ vs. $\mathrm{H}$ up to $90 \mathrm{kOe}$ at various temperature from $0.5 \mathrm{~K}$ to $300 \mathrm{~K}$ to a single curve representing Brillouin function variation for spin $S=1 / 2$. Also, the analysis of the variable frequency EPR line-shape and linewidth data in VOPc provides independent evidence for the presence of small exchange energy of the order of $0.1 \mathrm{~K}$.

\section{Acknowledgements:}

The authors (ZW and MSS) thank West Virginia University for the use of shared research facilities. A portion of this work was performed at the Steady High Magnetic Field Facilities, High Magnetic Field Laboratory, CAS, China. The high-field EPR measurements were made at the National High Magnetic Field Laboratory, which is supported by the National Science Foundation via Cooperative Agreement No. DMR-1157490, DOE and the State of Florida. 


\section{REFERENCES}

[1] N.B. Mckeown, Phthalocyanine Materials: Synthesis, Structure and Function, Cambridge, U.K.: Cambridge Univ. Press, 1998.

[2] J. E. Brumboiu, R. Totani, M. de Simone, M. Coreno, C. Grazioli, L. Lozzi, H.C. Herper, B. Sanyal, O. Eriksson, C. Puglia, B. Brena, Elucidating the 3d electronic configuration in manganese phthalocyanine. J. Phys. Chem. A. 118 (2014) 927-932.

http://pubs.acs.org/doi/abs/10.1021/jp4100747

[3] Z. Wang and M. S. Seehra, Ising-like chain magnetism, Arrhenius magnetic relaxation, and case against 3D magnetic ordering in $\beta$-Manganese phthalocyanine $\left(\mathrm{C}_{32} \mathrm{H}_{16} \mathrm{MnN}_{8}\right)$. J. Phys.: Condens. Matter. 28 (2016)136002/1-9. http://iopscience.iop.org/article/10.1088/0953$8984 / 28 / 13 / 136002$

[4] Z. Wang, K. L. Pisane, M.S. Seehra, Magnetic determination of the electronic state of Cu and exchange interactions in the $\alpha$ - and $\beta$-phases of molecular semiconductor copper phthalocyanine. IEEE Trans. Mag. 51 (2015) 2700104/1-4.

http://ieeexplore.ieee.org/xpl/articleDetails.jsp?arnumber=7112514

[5] M. Serri, W. Wu, L.R. Fleet, N.M. Harrison, C.F. Hirjibehedin, C.W.M. Kay, A.J. Fisher, G. Aeppli, S. Heutz, High-temperature antiferromagnetism in molecular semiconductor thin films and nanostructures. Nature Commun. 5 (2014) 3079/1-9.

http://www.nature.com/ncomms/2014/140121/ncomms4079/full/ncomms4079.html

[6] Z. Wang, M. Lee, E. S. Choi, J. Poston, and M. S. Seehra, Low temperature, high magnetic field investigations of the nature of magnetism in the molecular semiconductor $\beta$ - cobalt phthalocyanine $\left(\mathrm{C}_{32} \mathrm{H}_{16} \mathrm{CoN}_{8}\right)$. J. Magn. Magn. Mater. 407(2016) 83-86.

http://www.sciencedirect.com/science/article/pii/S0304885316300336

[7] H. Adler, M. Paszkiewicz, J. Uihlein, M. Polek, R. Ovsyannikov, T.V. Basova, T. Chasse and H. Peisert, Interface properties of VOPc on Ni (111) and grapheme/Ni (111): orientation- 
dependent charge transfer. J. Phys. Chem. C. 119 (2015) 8755-8762.

http://pubs.acs.org/doi/abs/10.1021/acs.jpcc.5b01485

[8] R.F. Ziolo, C.H. Griffiths, and J.M. Troup, Crystal structure of vanadyl phthalocyanine, phase II. J. Chem. Soc., Dalton Trans. (1980) 2300-2302.

http://pubs.rsc.org/en/Content/ArticleLanding/1980/DT/DT9800002300

[9] L. Li, Q. Tang, H. Li, and W. Hu, Molecular orientation and interface compatibility for high performance organic thin film transistor based on vanadyl phthalocyanine. J. Phys. Chem. B. 112 (2008) 10405-10410. http://pubs.acs.org/doi/abs/10.1021/jp800879g

[10] K. Eguchi, T. Takagi, T. Nakagawa, and T. Yokoyama, Molecular orientation and electronic states of vanadyl phthalocyanine on Si (111) and Ag (111) surfaces. J. Chem. Phys. C. 117 (2013) 22843-22851. http://pubs.acs.org/doi/abs/10.1021/jp406906k

[11] A.B.P. Lever, The magnetic behavior of transition-metal-phthalocyanines. J. Chem. Soc. (1965)1821-1829.

http://pubs.rsc.org/en/content/articlelanding/1965/jr/jr9650001821/unauth\#!divAbstract

[12] V. Narang, D. Korakakis, M.S. Seehra, Electronic state of Er in sputtered AlN:Er films determined by magnetic measurements. J. Appl. Phys. 116 (2014) 213911/1-6. http://scitation.aip.org/content/aip/journal/jap/116/21/10.1063/1.4903553

[13] D.G.E. Ingram and J. E. Bennett, Paramagnetic resonance in phthalocyanine, chlorophyll, and haemoglobin derivatives. J. Chem. Phys. 22 (1954) 1136-1137. http://scitation.aip.org/content/aip/journal/jcp/22/6/10.1063/1.1740286

[14] J. M. Assour, J. Goldmacher, and S.E. Harrison, Electron spin resonance of vanadyl phthalocyanine. J. Chem. Phys. 43 (1965) 159-165. http://scitation.aip.org/content/aip/journal/jcp/43/1/10.1063/1.1696446 
[15] M. Sato and T. Kwan, Electron spin resonance study of vanadyl phthalocyanine. J. Chem. Phys. 50 (1969) 558-559. http://scitation.aip.org/content/aip/journal/jcp/50/1/10.1063/1.1670852

[16] J. Krzystek, A. Ozarowski, and J.Telser, Multi-frequency, high-field EPR as a powerful tool to accurately determine zero-field splitting in hign-spin transition metal coordination complexes. Coord. Chem. Rev. 250 (2006) 2308-2324.

http://www.sciencedirect.com/science/article/pii/S0010854506000737

[17] J. Krzystek, S.A. Zvyagin, A. Ozarowski, S. Trofimenko, and J. Telser, Tunable-frequency, high-field electron paramagnetic resonance. J. Magn. Reson.178 (2006) 174-183.

http://www.sciencedirect.com/science/article/pii/S1090780705003095

[18] P. W. Anderson and P. R. Weiss, Exchange narrowing in paramagnetic resonance. Rev. Mod. Phys. 25 (1953) 269-276. https://journals.aps.org/rmp/abstract/10.1103/RevModPhys.25.269

[19] T. G. Castner and M. S. Seehra, Antisymmetric exchange and exchange-narrowed electronparamagnetic-resonance linewidths. Phys. Rev. B. 4 (1971) 38-45.

http://journals.aps.org/prb/abstract/10.1103/PhysRevB.4.38

[20] H. L. Retcofsky, G. P. Thompson, R. Raymond, and R. A. Friedel. Studies of e.s.r. linewidths in coals and related materials. Fuel. 54 (1975) 126-128. http://www.sciencedirect.com/science/article/pii/001623617590068X

[21] A. J. Henderson, Jr. and R. N. Rogers, Exchange and the " $10 / 3$ effect" in $\mathrm{K}_{2} \mathrm{CuCl}_{4} \cdot 2 \mathrm{H}_{2} \mathrm{O}$ and $\left(\mathrm{NH}_{4}\right)_{2} \mathrm{CuCl}_{4} \cdot 2 \mathrm{H}_{2} \mathrm{O}$. Phys. Rev. 152 (1966) 218-222.

http://journals.aps.org/pr/abstract/10.1103/PhysRev.152.218 